\title{
Conflitos e avanços na implementação de uma Residência Integrada em Saúde com ênfase em Cancerologia
}

\author{
Conflicts and advances in the implementation of a health \\ integrated residence program with emphasis in oncology
}

Rafael Bruno Silva Torres ${ }^{1}$, Ivana Cristina de Holanda Cunha Barreto², Juliana Burlamaqui Carvalho

${ }^{1}$ Programa de Pós-Graduação em Saúde Coletiva, Universidade Federal do Ceará (UFC) - Fortaleza (CE), Brasil.

2Fundação Oswaldo Cruz (FIOCRUZ Ceará) - Fortaleza (CE), Brasil.

${ }_{3}^{3}$ Hospital Universitário, Universidade Federal do Piauí (HU-UFPI) - Teresina (PI), Brasil.

DOI: http://dx.doi.org/10.7322/abcshs.v40i3.824

\begin{abstract}
RESUMO
Introdução: Neste artigo são apresentadas experiências obtidas em um estudo com residentes da primeira turma da Residência Integrada em Saúde-Cancerologia do Ceará, composta por 22 residentes multiprofissionais. Relato de experiência: Realizou-se um estudo de caso para proporcionar análise de um fenômeno contemporâneo no contexto em que ele ocorre, baseando-se em múltiplas fontes de evidência - análise bibliográfica e documental, grupos focais e análise fenomenológica. O material analisado gerou duas categorias principais: 1. "sobre os aprendizados e avanços" e 2. "sobre os conflitos e desafios". Percebe-se que os diferentes conhecimentos construídos pelos residentes continuam se complementando, permitindo que o campo da oncologia dialogue com outros saberes e produza novas reflexões. Os sujeitos estavam inseridos em um contexto real e complexo, com potencial crítico de repensar os lócus de sua inserção e propor mudanças. Conclusão: Compreende-se que todo o processo de ensino-aprendizagem em serviço traz em seu bojo desafios e dificuldades dos mais diversos e crescimentos pelas dificuldades, que podem ser pensadas como aprendizagens baseadas em problemas e mobilizadas pela prática. Considera-se que um melhor aproveitamento dos profissionais residentes precisa ser repensado como estratégia política e de formação no Sistema Único de Saúde (SUS), e a inserção das residências em saúde em espaços complexos, como os lócus de trabalho, não pode ser apenas naturalizada como espontânea ao longo dos anos, mas alvo de reflexão e problematização dos diversos atores envolvidos nesses processos.
\end{abstract}

Palavras-chave: educação em saúde; residência multiprofissional; serviço hospitalar de oncologia; pesquisa qualitativa.

\begin{abstract}
Introduction: In this article experiences are presented in a study with the first class of the Health Integrated Residence Program in Oncology of Ceará, Brazil, made up of 22 multiprofessional residents. Experience report: A case study was made to provide analysis of a contemporary phenomenon in the context in which it occurs, based on multiple sources of evidence - bibliographical and documentary analysis, focus groups and phenomenological analysis. The analyzed material generated two main categories: 1. "on learning and advances" and 2. "on conflicts and challenges." It is noticed that the different knowledge built by the residents continue to complement, allowing the field of oncology to dialogue with other knowledge and to produce new reflections. The subjects were placed in a real and complex context, with critical potential to rethink the locus of insertion and propose changes. Conclusion: It is understood that all service in teachinglearning process brings with it challenges and difficulties of the most diverse, and advances despite difficulties, which can be thought of as learning based on problems and mobilized by the practice. It considers that a better use of resident professionals needs to be rethought as a political strategy and training in Brazilian health system, and the insertion of health residences programs in complex spaces such as the locus of work can not only be naturalized as spontaneous over the years, but should be targeted for reflection and questioning of the various actors involved in these processes.
\end{abstract}

Keywords: health education; internship, nonmedical; oncology service, hospital; qualitative research. 


\section{INTRODUÇÃO}

O Sistema Único de Saúde (SUS) tem a competência de ordenar a formação na área da saúde ${ }^{1}$, o que cumpre "uma das mais nobres metas formuladas pela Saúde Coletiva no Brasil: tornar a rede pública de saúde uma rede de ensino-aprendizagem no exercício do trabalho"2. Para tanto, o Ministério da Saúde (MS) tem desenvolvido estratégias variadas e políticas voltadas para a adequação da formação e qualificação dos trabalhadores da área da saúde às reais necessidades da população e ao desenvolvimento do próprio SUS ${ }^{3}$.

A Educação Permanente em Saúde (EPS) aparece como uma alternativa viável a realização desses objetivos de qualificar e atender às demandas do SUS. Esse tem sido o enfoque educacional reconhecido como o mais apropriado para produzir transformações nas práticas e nos contextos de trabalho, fortalecendo a reflexão na ação, o trabalho em equipes e a capacidade de gestão sobre os processos locais ${ }^{4}$.

Por EPS entende-se "o conceito pedagógico para efetuar relações orgânicas entre ensino e as ações e serviços, e entre a docência e atenção à saúde" ${ }^{\text {. }}$. As Residências Multiprofissionais em Saúde (RMS) representam um dos eixos de ação da Política Nacional de Educação Permanente em Saúde (PNEPS) ${ }^{6}$. No total, são 14 profissões de saúde que têm nas RMS formas de acesso ao trabalho e à aprendizagem no SUS7 .

O Instituto Nacional de Câncer José Alencar Gomes da Silva (INCA) é o centro de referência no Brasil em tratamento e educação na área oncológica e, atendendo a disposições legais do MS, em 2010 instituiu o primeiro programa de RMS em Oncologia, buscando elaborar um currículo que articulasse os saberes de diversas categorias profissionais, baseado na integralidade do cuidado e sob uma abordagem interdisciplinar ${ }^{8}$.

Tal programa procurou se nortear pelas diretrizes da PNEPS, tratando da forte relação entre assistência, ensino, pesquisa e gestão. Desde então, todos os programas em oncologia buscam trabalhar nesses preceitos, tentando ainda vencer alguns desafios, tais como: a implementação de um modelo de ensino que articule teoria e prática; a superação de um modelo disciplinar fragmentado, tendo a interdisciplinaridade e a integralidade como eixos transversais; e a concepção de avaliação como processual e inclusiva ${ }^{8}$.

Estima-se que atualmente no Brasil existem entre 10 e 15 programas de RMS com ênfase na atenção à pessoa com câncer - sendo a maioria na região Sudeste. No Ceará, a Residência Integrada em Saúde (RIS) com ênfase em Cancerologia, proposta pela Escola de Saúde Pública do Ceará (ESP-CE), teve início em 2013 e foi criada com o objetivo de formar lideranças técnicas, científicas e políticas para a qualificação da linha de cuidado da pessoa com câncer, visando, ainda, à promoção, à proteção e à recuperação da saúde, baseando-se na colaboração interprofissional, na integralidade e na intersetorialidade ${ }^{9}$.

A partir da colaboração no programa RIS-Cancerologia, surgiu o interesse em investigar como os residentes da primeira turma vivenciaram seu processo de residência, o que aprenderam e quais foram seus principais desafios. Nesse sentido, o objetivo principal deste artigo é compreender os principais conflitos e avanços vivenciados pelos residentes multiprofissionais da primeira turma da RIS-Cancerologia.

\section{RELATO DE EXPERIÊNCIA}

Realizou-se um estudo de caso, utilizando-se de diversas opções metodológicas para complementar a investigação - análise bibliográfica e documental, grupos focais e análise fenomenológica. O estudo de caso é uma investigação empírica que proporciona analisar um fenômeno contemporâneo no contexto em que ele ocorre, baseando-se em múltiplas fontes de evidência, o que traz a necessidade de triangulação dos resultados, com o intuito de confirmar a sua validade ${ }^{10}$.

Esse tipo de estudo se beneficia de diversos referenciais teóricos que embasam a coleta e a análise de dados, e a sua triangulação permite uma confrontação de achados e teorias, em múltiplas combinações, que validam os resultados e aumentam a credibilidade das interpretações ${ }^{10}$.

Entendendo que desde o princípio a grande proposta da RIS foi a integração, optou-se por utilizar o grupo focal como ferramenta para coleta dos dados principais, visando promover uma possibilidade de diálogo entre os próprios residentes multiprofissionais. Entende-se que o grupo focal funciona como uma espécie de entrevista grupal, que tem seus fundamentos teórico-metodológicos nas Teorias de Grupo, na Sociologia e na Psicologia Social Crítica ${ }^{11}$.

A primeira turma da RIS-Cancerologia era composta, no total, por 22 residentes multiprofissionais de 6 categorias profissionais distintas (Enfermagem, Farmácia, Fisioterapia, Nutrição, Psicologia e Serviço Social). Foram realizados cinco grupos focais, nos quais os sujeitos participantes foram divididos de forma aleatória. No total, 12 residentes multiprofissionais participaram, a média de duração de cada grupo foi de 50 minutos e todos foram realizados em local que resguardou a privacidade. Os áudios foram gravados para posterior transcrição e análise, e cada grupo contou com um moderador - responsável pela pesquisa - e um observador, convidado externo que auxiliou na análise da interação grupal.

Como ferramenta de análise e tratamento dos dados, buscou-se uma aproximação com a análise fenomenológica, com base na fenomenologia de Merleau-Ponty, a partir de considerações trazidas por Moreira $^{12}$, para o qual compreender significa distinguir a singularidade de cada acontecimento e seus significados.

Os seguintes passos para a compreensão da experiência foram utilizados: divisão do texto nativo (transcrição literal das entrevistas) em movimentos (espécies de categorias), seguida por uma análise descritiva dos significados emergentes no movimento e discussão no "sair dos parênteses", em que a experiência se encontra com a teoria, mas sempre buscando o significado vivido da experiência ${ }^{12}$. Os movimentos serão apresentados respeitando a ordem de expressão das vivências, levando o leitor a aproximar-se dessa realidade ${ }^{13}$. 
O trabalho seguiu as orientações da Resolução n 466/2012, do Conselho Nacional de Ética em Pesquisa, que orienta sobre a pesquisa envolvendo seres humanos ${ }^{14}$, e foi aprovado com o Parecer 965.661 no Comitê de Ética em Pesquisa do Instituto do Câncer do Ceará - hospital ao qual os residentes estiveram vinculados.

De modo que resguarde a identidade dos sujeitos colaboradores, as falas dos 12 residentes foram nomeadas aleatoriamente de $\mathrm{R} 1$ a R12, os grupos focais realizados foram numerados de G1 a G5, de acordo com a ordem de ocorrência, e as profissões não foram identificadas na transcrição.

A opção por não identificar as profissões, mas denominá-las em núcleos, vem da existência de uma inevitável sobreposição de limites entre as disciplinas, de modo que a institucionalização dos saberes e sua organização em práticas se dá na confrontação entre núcleos e campos. Núcleo como uma aglutinação de conhecimento, demarcando a identidade de uma área de saber; e campo como um espaço de limites imprecisos, no qual cada profissão busca em outras o apoio para cumprir suas tarefas e práticas ${ }^{15}$.

Dos principais movimentos emergidos do texto nativo, foram elencadas duas categorias principais: 1 . "sobre os aprendizados e avanços" e 2. "sobre os conflitos e desafios". Essas categorias serão mais bem compreendidas nos próximos parágrafos, bem como explicitadas em suas várias formas e significados de aparecimento.

\section{"Me deu uma série de habilidades que antes eu não tinha": sobre os aprendizados e avanços}

Mostra-se fundamental observar os aprendizados obtidos com a RMS, entendendo que o trabalho na saúde requer trabalhadores que aprendam a aprender; práticas cuidadoras; desenvolvimento de si, dos coletivos institucional e político da saúde; além da implicação com as práticas concretas de cuidado às pessoas e às coletividades, no ensino e na produção de conhecimento ${ }^{2}$.

R6G2: [...] se eu for pensar hoje tudo que eu aprendi ao longo desses dois anos, foi muita coisa, né? A segurança que eu tenho hoje, de começar a trabalhar no hospital, é diferente!

R7G2: [...] E você com pouca bagagem profissional, com relativa bagagem teórica também, mas com pouca bagagem profissional, você ter que dar conta e você ir aprendendo, ir errando e aprendendo, então isso pra mim foi um aprendizado muito bom, né!

\section{"Meus aprendizados marcantes": a aprendizagem integrada}

De todas as aprendizagens vividas, aquela que se sobressai como mais emergente nas falas dos residentes é sobre o aprendizado entre as profissões e o trabalho em equipe.

R1G1: [...] acho que esteve muito nos meus aprendizados marcantes, é a questão do trabalho em equipe, eu lembro que antes de fazer a residência eu pensava: por que que os médicos têm uma residência só deles e a nossa é todo mundo junto? Isso é com certeza uma coisa para economizar né... então, pra mim, antes, o fato de ser multiprofissional era um fator meio que desqualificante, como se fosse menor do que eu ter residência em [núcleo], e hoje eu tenho outra visão, eu acho que o ideal seria que em um determinado momento do mundo, que eu não vejo chegar ou estar tão próximo, que todas as residências fossem multi, que todas elas fossem conjuntas!

A formação profissional para a área da saúde faz referência à formação de profissionais generalistas, com visão humanista, crítica e integradora, capazes de atuar com qualidade e resolubilidade no SUS ${ }^{2}$. Todo processo que esteja comprometido com a EPS pretende gerar no trabalhador, no seu cotidiano de produção do cuidado em saúde, transformações da sua prática — o que implicaria força de produzir e capacidade de problematizar a si mesmo no agir ${ }^{16}$.

A vivência possibilitada na RMS busca englobar todos esses aspectos, além de favorecer a busca pela integralidade em saúde. Participar de um processo formativo que se baseia na construção articulada de diferentes profissões da saúde, buscando construir um saber comum, no qual se agreguem as contribuições dos diferentes núcleos profissionais, pode efetivar uma prática renovadora ${ }^{17}$.

Uma estratégia da RIS para disparar mudanças foi a inserção de equipes multiprofissionais de residentes em todos os cenários de prática. As equipes multiprofissionais permitem uma mediação de sabres e competências na construção de projetos terapêuticos, tendo a gestão colegiada do processo de cuidado e superando o tradicional modelo biomédico ${ }^{18}$. Pela fala dos residentes multiprofissionais, fica clara uma efetiva prática integrada dentro das equipes e a riqueza que isso possibilita tanto no ato do cuidado em saúde como no processo de trabalho-aprendizagem.

\section{"É fun-da-men-tal": a importância do preceptor de campo}

Como forma de efetivar o aprendizado integrado, os residentes discutiram sobre a importância do preceptor de campo, figura-chave no processo de integração entre as profissões. O preceptor de campo tem a função de supervisão docente-assistencial no campo de aprendizagens profissionais, deve estar diariamente presente nos ambientes onde se desenvolvem as aprendizagens em serviço, facilitando o desenvolvimento de competências comuns da ênfase e da saúde ${ }^{19}$.

R4G1: [...] o preceptor de campo ainda é fun-da-men-tal se quer implementar o que está escrito no projeto.

R1G1: Uma das dificuldades às vezes pro hospital entender a necessidade do preceptor de campo é porque eu vejo que é muito recorrente a compreensão aqui de que, a partir do momento 
que eu tenho a equipe, que tem profissionais de várias categorias diferentes, essa equipe naturalmente tem um trabalho interdisciplinar, multiprofissional... e, assim, muitas vezes isso acontece de uma forma supercompartimentalizada, não é você só colocar as pessoas juntas que o trabalho vai estar integrado, e às vezes... eu sinto isso... que é como se o hospital: "mas já tem todo mundo, então já funciona assim”. Não! Não funciona assim, né... funciona cada um no seu cantinho! E por isso a importância de ter pessoas que estejam ali com a função de chamar essa integração.

A figura do preceptor de campo surgiu a partir do preceptor de território, que tem a responsabilidade pelo processo de EPS dos profissionais nos territórios de saúde da família. A concepção da preceptoria de território tomou forma por meio da ação multiprofissional, tendo como função trabalhar com a formação dos residentes nas equipes de saúde, organizar os serviços e promover a gestão participativa nos territórios ${ }^{20}$.

Preceptoria de campo foi, portanto, uma adaptação possível ao "território hospitalar", que, mesmo com suas especificidades e diferenças de um contexto comunitário de atuação, precisa potencializar a integração profissional - além de uma referência a ideia de núcleo e campo de conhecimento ${ }^{15}$. A fala dos residentes foi bem clara ao salientar a importância dessa figura no processo de integração das equipes.

\section{"O cenário foi dado": o projeto RIS e a formação para assistência, gestão e ensino}

Os residentes multiprofissionais discutiram sobre os objetivos do projeto RIS de formar profissionais para tornarem-se "lideran-

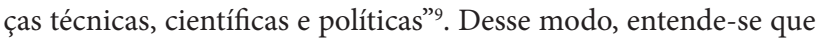
o desejo do projeto era preparar os profissionais para assumirem espaços de assistência nos serviços de saúde, gestão de programas e políticas, além de serem atores na construção do conhecimento em Cancerologia.

R1G1: [...] talvez aquilo que foi mais difícil pra mim foi também onde eu mais aprendi, que foi nessa questão das relações institucionais, e acho que isso passa muito por desenvolver habilidades relacionadas à gestão. [...] as possibilidades para desenvolver as três coisas aconteceram.

R8G2: Eu acho que de gestão, pelo menos falando em relação ao que eu vivenciei, eu acho que não foi favorável, pelo contrário, eu acho que a gente viu como se fosse um boicote em relação a isso, a questão de liderança e a questão de gestão, de que existia um funcionamento e precisamos cumprir como é o funcionamento do hospital, da rede e tal. Então essa questão de gestão e liderança pra mim não aconteceu!

A RMS é uma prática impulsionadora de melhorias na saúde, pautada numa pedagogia dialógica e problematizadora.
Vem tentar estabelecer espaços cotidianos de reflexão coletiva sobre a prática tencionando a construção da cogestão dos processos de trabalho. A RMS tem um caráter indutor de mudanças no campo do fazer-saber em saúde, a partir da criação e do fortalecimento de espaços de problematização e diálogo sobre as práticas ${ }^{21}$. A RIS propôs espaços significativos e coerentes para o desenvolvimento de competências, por meio de momento teóricos, estágios com rodízio em rede e rodas de discussões com frequência regular. Compreende-se que os objetivos se mostraram amplos e representaram diferentes variáveis do processo saúde-doença.

A partir desses espaços indutores de transformação, os residentes reconhecem que as possibilidades de assistência, ensino e gestão foram dadas; contudo, se percebe a existência de fatores individuais, que influenciam a diversidade de interesses e potencialidades. Os diversos espaços produzidos pela residência revelaram as micropolíticas das relações. As relações de poder parecem ter repercutido na forma como foi vivenciado o aspecto relacionado à gestão. As discussões trazidas demonstram a vivência dessas relações, que, mesmo sendo percebidas como um "boicote", favoreceram o desenvolvimento de habilidades relacionadas à gestão.

\section{"Uma série de sobrecargas em cima de você": sobre os conflitos e desafios}

Os participantes discorreram livremente sobre os principais desafios vivenciados durante a residência multiprofissional, sua vivência das dificuldades e como foram, ou não, superadas.

R4G4: [...] Uma série de sobrecargas em cima de você, por exemplo, hoje; hoje tem a sobrecarga do trabalho de conclusão da residência, tem a sobrecarga do setor, porque se a [profissional] não está no setor, quem responde pelo setor sou eu. Aí fica aquela pressão. Ficam todos os tipos de pressão em cima de você.

R2G4: Eu acho que a dificuldade forçou a ter um aprendizado, a buscar um aprendizado!

R5G4: Forçou a aprender, forçou a conseguir se relacionar dentro desse fluxo de poder, de como tudo funciona, desse fluxo de relacionamentos.

Todo processo de ensino-aprendizagem traz em seu bojo desafios e dificuldades dos mais diversos. $\mathrm{O}$ crescimento pelas dificuldades pode ser pensado como aprendizagens baseadas em problemas, mobilizadas pelo cotidiano e pela prática. Isso demonstra que esses sujeitos estavam inseridos em um contexto real e complexo, no qual tudo acontecia ao mesmo tempo.

Na experiência das RMS em uma instituição hospitalar, é difícil para muitos profissionais integrar ações de assistência, ensino, pesquisa e gestão, visto que a assistência continua sendo a prioridade dos hospitais como prestadores de atendimento à saúde ${ }^{22}$. A implementação de programas de RMS, especialmente nos hospitais, precisa ser constantemente avaliada e repensada. 


\section{"Não tem uma especialidade": o reconhecimento na Oncologia}

O reconhecimento de cada profissão na Oncologia foi discutido. Apesar da importância epidemiológica que o câncer vem apresentando em nível mundial, e do respaldo das políticas nacionais ${ }^{6,23,24}$, algumas profissões da saúde ainda não possuem um reconhecimento efetivo dentro da Cancerologia, o que se mostra um desafio cultural ainda a ser superado.

R2G1: Para o [meu núcleo] já existe uma especialidade. [...] Durante esse percurso, eu percebi que seria uma vantagem porque seria uma especialidade e acabar saindo daqui já tendo, sem precisar correr atrás de fazer uma pós à parte.

R8G2: [...] acho que do próprio reconhecimento da sociedade, é uma coisa mesmo cultural que não vai mudar do dia para a noite, não é uma coisa que, sei lá, a gente possa resolver agora, mas tem essa dificuldade mesmo de postos de trabalho.

Mesmo que a atuação de cada profissão na área seja de extrema relevância no cuidado ao paciente com câncer, para alguns núcleos, na graduação ou em contextos de trabalho, ainda existem poucos espaços disponíveis para atuação e discussão. Outros núcleos profissionais, por sua vez, já reconhecem essa possibilidade, inclusive legalmente, a partir de seus conselhos de representação profissional.

\section{"Revela muito do que é hegemônico, né?!": crítica a um modelo biologicista}

Os participantes discutiram sobre o modelo clássico de pensamento construído na atuação em saúde e os impactos que esse modelo trouxe a sua vivência.

R1G1: [...] Por que é que se entende que eu [núcleo] saber de quimioterapia é mais importante do que o [núcleo] saber dos direitos do paciente ou dos impactos psicológicos de uma hospitalização prolongada?

R8G2: Prevalecia o saber médico, o que é que ia ser feito com aquele paciente, que cirurgia, que procedimento, e os outros profissionais eram como se fossem assim... né... por exemplo, a sensação que a gente tinha era como se fôssemos menos relevantes, porque às vezes não existia nem espaço pra falar.

O processo tradicional de ensino-aprendizagem em saúde tem origem no modelo flexneriano, introduzido no Brasil por volta de 1940 e com um forte viés biomédico e biologicista, o que, historicamente, fomenta uma lógica de fragmentação e superespecialização - especialmente no contexto hospitalar. Nos últimos anos, tem se enfatizado a colaboração entre as profissões de saúde como tentativa de superação de diversos impasses observados que prejudicam a qualidade e a resolutividade das práticas de assistência, não incorporando a dimensão da integralidade ${ }^{25}$.
A especialização exacerbada marca a área da Oncologia, fortalece a medicina dos órgãos e a visão organicista da doença que deve ter seus limites reconhecidos ${ }^{26}$. É importante que ocorra uma desvinculação da formação em saúde de um caráter unicamente biologicista e individualista, que ignora outras áreas do conhecimento. Nesse sentido, compreende-se que a complexidade do fenômeno saúde-doença em Oncologia requer, cada vez mais, uma perspectiva interdisciplinar ${ }^{18,25}$.

\section{"Antes não existia, a gente foi e ocupou o espaço": o desafio da primeira turma}

Ser a primeira turma de um programa dessa magnitude tem suas vantagens e desvantagens. Os residentes puderam vivenciar isso ao longo dos dois anos e apresentaram suas considerações:

R7G2: [...] a gente sabe o que a gente sofreu, a gente sabe o que a gente chorou, a gente sabe que a gente se estressou, que a gente se aborreceu; quantas vezes a gente teve vontade de desistir, mas foi indo... indo... e passando por tudo isso e acumulando estresse e coisa. Mas isso realmente foi um processo de construção, que por vezes foi doloroso, que por vezes foi mais tranquilo, mas se hoje eles veem e recebem essa equipe mais acessível pro trabalho multiprofissional, foi por conta de muita coisa que a gente realmente construiu mesmo!

R11G5: Não tinha um referencial. Então a gente acaba que ficava no meio dessa discussão, as vezes pendendo pra um lado, as vezes pendendo pro outro.

O pioneirismo vem acompanhado de novidades e resistências à mudança. Certos sujeitos e grupos apoiaram as novidades trazidas pela RIS, enquanto outros sentiram receio, acostumados aos processos anteriores. A importância de investigar a primeira turma se encontra justamente nas considerações que esses sujeitos têm a fazer sobre sua experiência pioneira. Efetivações e mudanças das turmas seguintes vão ocorrer ao longo do projeto RIS como um todo, mas muitos aspectos foram e serão efetivados a partir da primeira experiência.

\section{"Pra onde é que vai todas essas pessoas?": reflexão sobre o número de vagas}

Em 2013 houve uma significativa ampliação nas vagas de RMS no Estado do Ceará - por meio da implementação da RIS-ESP-CE, foram 222 vagas distribuídas em todo o Estado, nas ênfases: Cancerologia; Saúde Mental Coletiva; Saúde da Família e Comunidade; e Saúde Coletiva ${ }^{9}$. Nos anos seguintes, essas vagas foram ampliadas ${ }^{27}$. Os participantes levantaram questionamentos importantes sobre esses números:

R4G1: É a política de saúde que não dá conta, ela não tem uma continuidade. Então é um governo que investe em ti por dois anos, pra estudar, pra se especializar em Oncologia; quando tu chega lá, tu está altamente especializado, aí você sai e você vai procurar [...]. Se vira pra procurar emprego e vai se inserir em outras coisas. 
R1G1: É uma pauta nacional da residência, essa questão da absorção na perspectiva do residente, do retorno de trabalho. Mas na perspectiva da residência, como política, de ser um investimento público nacional que tenha um retorno.

Em discussões realizadas pelo Fórum Nacional de Residentes em Saúde (FNRS), foi produzida a Carta de Fortaleza, no ano de 2013, que, entre outros aspectos, discutiu sobre a necessidade da criação de uma política nacional de residências integrada, que dialogue e respeite as necessidades locais, construída de maneira democrática e participativa por todos os atores que constroem as residências. É importante a articulação com a PNEPS, respeitando a absorção do residente pelo SUS por meio de concurso público, ressaltando o investimento público feito na formação desses profissionais, sendo a residência parte integrante da carreira no SUS ${ }^{28}$.

A pauta sobre a absorção desses profissionais pelo SUS é nacional e acontece desde os primeiros programas de RMS implementados no Brasil. $\mathrm{O}$ atual contexto, com variados programas e diversas vagas, reafirma a importância dessa questão e traz à tona a necessidade de uma política nacional e longitudinal que efetive a absorção desses profissionais capacitados pelo SUS para que atuem efetivamente nesse sistema.

\section{DISCUSSÃO}

Compreender como foi o processo vivenciado pela primeira turma da RIS-Cancerologia pode favorecer possíveis alterações no desenho metodológico das turmas seguintes, bem como favorecer o desenvolvimento de outros programas.
A inserção das RMS em espaços complexos como os lócus de trabalho não pode ser apenas naturalizada como espontânea ao longo dos anos, mas deve ser alvo de reflexão e problematização das instituições executoras e formadoras e de políticas públicas, em parceria com os diversos atores envolvidos no processo e com base em novas reflexões produzidas por aqueles grupos.

Este trabalho possibilitou a percepção de que os diferentes conhecimentos construídos pelas diversas especialidades se complementam, permitindo que o campo da Oncologia dialogue com outros saberes. Podemos pensar em um olhar para a especialidade diferente do trazido pela lógica positivista. Nas RMS, confirma-se que a especialização em saúde exige domínio de aspectos de núcleo e campo, que se interligam contínua e complexamente.

Por fim, um melhor aproveitamento desses profissionais precisa ser repensado como estratégia política e de formação no SUS. Com tantas vagas ofertadas, tantas ênfases e programas em execução, cabem os questionamentos: que espaços futuros esses profissionais ocuparão? E, após dois anos de intenso aprimoramento de competências e qualificação, como eles poderão ser absorvidos pelo SUS? Para assim, finalmente, efetivar um dos objetivos fundamentais da RMS: a melhoria dos desenhos técnico-assistenciais dos serviços de saúde.

\section{AGRADECIMENTOS}

Aos residentes multiprofissionais que compuseram a primeira turma da RIS-ESP-CE Cancerologia e a Aline Luiza de Paulo Evangelista, mestranda do Programa de Pós-Graduação em Saúde Coletiva da Universidade Federal do Ceará (UFC), pela contribuição como observadora dos grupos focais.

\section{REFERÊNCIAS}

1. Brasil. Senado Federal. Constituição da República Federativa do Brasil. Brasília: Senado; 1988

2. Ceccim RB. Educação permanente em saúde: descentralização e disseminação de capacidade pedagógica na saúde. Ciênc Saúde Coletiva. 2005;10(4):219-30. http://dx.doi.org/10.1590/S1413-81232005000400020

3. Brasil. Ministério da Saúde. Política Nacional de Educação Permanente em Saúde. Brasília: Ministério da Saúde; 2009.

4. Davini MC. Enfoques, problemas e perspectivas na educação permanente dos recursos humanos de saúde. In: Brasil. Política Nacional de Educação Permanente em Saúde. Brasília: Ministério da Saúde; 2009. p.39-56.

5. Brasil. Ministério da Saúde. Portaria MS n 1996, de 20 de agosto de 2007. Dispõe sobre as diretrizes para a implementação da Política Nacional de Educação Permanente em Saúde e dá outras providências. Brasília: Ministério da Saúde; 2007.

6. Brasil. Ministério da Saúde. Portaria $n^{\circ} 198$, de 13 de fevereiro de 2004. Institui a Política Nacional de Educação Permanente em Saúde como estratégia do Sistema Único de Saúde para a formação e o desenvolvimento de trabalhadores para o setor e dá outras providências. Brasília: Ministério da Saúde; 2004.
7. Brasil. República Federativa do Brasil. Portaria Interministerial MS/ MEC $n^{\circ}$ 45, de 12 de janeiro de 2007. Dispõe sobre a Residência Multiprofissional em Saúde e a Residência em Área Profissional da Saúde e institui a Comissão Nacional de Residência Multiprofissional em Saúde. Brasília: Ministério da Saúde/Ministério da Educação; 2007.

8. Brasil. Plano de curso do programa de residência multiprofissional em oncologia. 2 ed. Rio de Janeiro: Instituto Nacional de Câncer José Alencar Gomes da Silva; 2014.

9. Governo do Estado do Ceará. Secretaria da Saúde do Estado do Ceará. Escola de Saúde Pública do Ceará. Residência Integrada em Saúde. Fortaleza: folheto; 2013

10. Yin RK. Estudo de caso: planejamento e métodos. 3 ed. Porto Alegre: Bookman; 2009.

11. Kind L. Notas para o trabalho com a técnica de grupos focais. Psic Rev. 2004;10(15):124-36.

12. Moreira V. O método fenomenológico de Merleau-Ponty como ferramenta crítica na pesquisa em psicopatologia. Psicol Reflex Crít. 2004;17(3):447-56. http://dx.doi.org/10.1590/S0102-79722004000300016

13. Holanda A. Questões sobre pesquisa qualitativa e pesquisa fenomenológica. Anal Psicol. 2006;24(3):363-72. 
14. Brasil. Ministério da Saúde. Conselho Nacional de Saúde. Resolução CNS 466, de 12 dez 2012. Dispõe sobre as diretrizes e normas regulamentadoras de pesquisas envolvendo seres humanos. Brasília: Ministério da Saúde; 2012

15. Campos GWS. Saúde pública e saúde coletiva: campo e núcleo de saberes e práticas. Ciênc Saúde Coletiva. 2000;5(2):219-30. http://dx.doi.org/10.1590/S1413-81232000000200002

16. Merhy EE. O desafio que a educação permanente tem em si: a pedagogia da implicação. Interface. 2005;9(16):172-74. http://dx.doi.org/10.1590/S1414-32832005000100015

17. Salvador AS, Medeiros CS, Cavalcanti PB, Carvalho RN Construindo a multiprofissionalidade: um olhar sobre a residência multiprofissional em saúde da família e comunidade. Rev Bras Cienc Saúde. 2011;15(3):329-38 http://dx.doi.org/10.4034/RBCS.2011.15.03.08

18. Furtado JP. Equipes de referência: arranjo institucional para potencializar a colaboração entre disciplinas e profissões. Interface. 2007;11(22):239-55 http://dx.doi.org/10.1590/S1414-32832007000200005

19. Governo do Estado do Ceará. Secretaria da Saúde do Estado do Ceará. Comissão de Residência Multiprofissional em Saúde da Escola de Saúde Pública do Ceará. Regimento Interno. Fortaleza: s.d.

20. Pagani R, Andrade LOM. Preceptoria de território, novas práticas e saberes na estratégia de educação permanente em saúde da família: o estudo do caso de Sobral, CE. Saúde Soc. 2012;21(Supl 1):94-106.

http://dx.doi.org/10.1590/S0104-12902012000500008

21. Carvalho AB, Nepomuceno LB. A residência multiprofissional em saúde da família potencializando a transformação através da formação: uma utopia necessária. Sanare. 2008;7(2):31-7.
22. Baldisserotto J, Falardo AP, Pasini VL, Schmidt MH, Azeredo $N$, Martins AR, et al. Residência Integrada em Saúde do Grupo Hospitalar Conceição RIS/GHC: uma estratégia de desenvolvimento de trabalhadores para o SUS. In: Residência Multiprofissional em Saúde: experiências, avanços e desafios. Brasília: Ministério da Saúde; 2006. p.355-74.

23. Brasil. Ministério da Saúde. Portaria MS n 2439, de 08 de dezembro de 2005. Institui a Política Nacional de Atenção Oncológica: promoção, prevenção, diagnóstico, tratamento, reabilitação e cuidados paliativos a ser implantada em todas as unidades federadas, respeitadas as competências das três esferas de gestão. Diário Oficial da União. 2005;236(1):80-1.

24. Brasil. Portaria MS n 874, de 16 de maio de 2013. Institui a Política Nacional para a prevenção e controle do câncer na rede de atenção à saúde das pessoas com doenças crônicas no âmbito do Sistema Único de Saúde (SUS). Brasília: Ministério da Saúde; 2013.

25. Ceccim RB, Feuerwerker LCM. Mudança na graduação das profissões de saúde sob o eixo da integralidade. Cad Saúde Pública. 2004;20(5):1400-10.

http://dx.doi.org/10.1590/S0102-311X2004000500036

26. Barreto ICHC, Andrade LOM, Loiola F, Paula JB, Miranda AS, Goya N. Educação Permanente e a Construção de Sistemas Municipais de Saúde Escola: o caso de Fortaleza (CE). Divulg Saúde Debate. 2006;(34):31-46

27. Governo do Estado do Ceará. Secretaria da Saúde do Estado do Ceará. Escola de Saúde Pública do Ceará. Edital nº 01/2014. Fortaleza: 2014

28. Fórum Nacional de Residentes em Saúde (FNRS). Carta de Fortaleza. Disponível em: https://drive.google.com/file/d/OBkqN1hIN6M4dm1iSjc0WXV1OVE/view. Acesso em: 06 maio 2015. 